\title{
POINTWISE CONVERGENCE OF THE FOURIER TRANSFORM ON LOCALLY COMPACT ABELIAN GROUPS
}

\author{
Maria L. Torres de Squire*
}

\begin{abstract}
We extend to locally compact abelian groups, Fejer's theorem on pointwise convergence of the Fourier transform. We prove that $\lim \varphi \varphi_{U} f(y)=f(y)$ almost everywhere for any function $f$ in the space $\left(L^{P}, l^{\infty}\right)(G)$ (hence in $\left.L^{P}(G)\right), 2 \leq p \leq \infty$, where $\{\varphi U\}$ is Simon's generalization to locally compact abelian groups of the summability Fejer Kernel. Using this result, we extend to locally compact abelian groups a theorem of $F$. Holland on the Fourier transform of unbounded measures of type $q$.
\end{abstract}

\section{Notation and Preliminary Results}

Throughout, $G$ is a locally compact abelian group, with dual group $\Gamma$, and Haar measure $m$. By the structure theorem, $G$ is represented by $\mathbf{R}^{a} \times G_{1}$, where $a$ is a nonnegative integer and $G_{1}$ is a group which contains an open compact subgroup $H$. The set of basic neighbourhoods of $x \varepsilon G$ is denoted by $\mathcal{N}_{x}(G)$. We write $C_{c}(G), C_{0}(G)$ for the spaces of functions on $G$ that are continuous, with compact support and vanish at infinity, respectively. We consider the amalgam spaces $\left(L^{p}, l^{q}\right)(G),\left(C_{0}, l^{q}\right)(G)(1 \leq p, q \leq \infty)$ as defined in [S]. The Fourier transform (inverse Fourier transform) of a measure $\mu$, is denoted by $\hat{\mu}(j)$. We let $A_{c}(G)$ be the set of all functions $f$ in $C_{c}(G)$ such that $\hat{f} \varepsilon L^{1}(\Gamma)$. The characteristic function of a subset $E$ of $G$ is denoted by $\chi_{E}$. The conjugate $p^{\prime}$ of a number $p$ is such that $1 / p+1 / p^{\prime}=1$. For each $U \in \mathcal{N}_{0}(G)$, A.B. Simon [Si] defined a function $\varphi_{U}$ as the product of two functions $\alpha_{U}$ and $\beta_{U}$ defined on $\mathrm{R}^{a}$ and on $G_{1}$, respectively.

*1980 Mathematics Subject Classification (1985 Revision). Primary 43A55, 43A25.

Research supported by NSERC grant 7914. 
The function $\beta_{U}$ is continuous, nonnegative, with $L^{1}(G)$-norm equal to 1 , and

$$
\sup _{G}\left|\beta_{U}(x)\right|=B_{U} \leq 2 m(U) / 1-2 m(U) \quad \text { finite. }
$$

Hence $B_{U} \rightarrow 0$ as $U \rightarrow 0$.

The function $\alpha_{U}$ is defined as follows.

Let $\left(-\delta_{1}, \delta_{1}\right) \times \cdots \times\left(-\delta_{a}, \delta_{a}\right) \times U_{H}$ be a product neighbourhood contained in $U$, where $\delta_{i}>0 \quad(i=1, \ldots, a)$, and $U_{H}$ is an element of $\mathcal{N}_{0}(G)$ included in $H$. For $i=1, \ldots, a$ we set $U_{i}=\left(-\delta_{i}, \delta_{i}\right), N_{i}=1 / \delta_{i}$, and define the function $\alpha_{U_{i}}$ on $\mathbf{R}$ by

$$
\alpha_{U_{i}}(t)=\frac{1-\cos \left(N_{i} t\right)}{\pi N_{i} t^{2}}
$$

For $t=\left(t_{1}, \ldots, t_{a}\right)$ in $\mathbf{R}^{a}$, the function $\alpha_{U}$ is given by $\alpha_{U}(t)=$ $\Pi_{i=1}^{a} \alpha_{U_{i}}\left(t_{i}\right)$. Clearly $\alpha_{U}$ is continuous, nonnegative, and its $L^{1}\left(\mathbf{R}^{a}\right)$-norm is equal to 1. Each $\varphi_{U}$ has the following properties. For a proof see [Si].

1.1) $\varphi_{U}$ is continuous, nonnegative and bounded

1.2) $\varphi_{U}$ is integrable and $\left\|\varphi_{U}\right\|_{1}=1$

1.3) $\hat{\varphi}_{U} \varepsilon C_{c}(\Gamma)$ and $\left\|\hat{\varphi}_{U}\right\|_{\infty} \leq 1$

1.4) $\varphi v(x)=\int_{\Gamma} \hat{\varphi}(\gamma) \gamma(x) d \gamma$ by 1.3$)$

1.5) For $\varepsilon>0$ and $U \varepsilon \mathcal{N}_{0}(G)$ given, we can find a $V$ such that if $V^{\prime} \leq V$ and $x \notin \quad U$, then $\varphi_{V}(x)<\varepsilon$ and $\int_{G-V} \varphi_{V^{\prime}}(x) d x<\varepsilon$.

1.6) $\lim _{U} \hat{\varphi}_{U}(\gamma)=1$.

1.7) the family $\left\{\varphi_{U} \mid U \varepsilon \mathcal{N}_{0}(G)\right\}$ is an approximate identity in $L^{1}(G)$.

We add to this list the fact that each $\varphi_{U}$ belongs to the Wiener algebra $\left(C_{0}, l^{1}\right)(G) \quad[W]$

Proposition 1.1. For each $U$ in $\mathcal{N}_{0}(U)$, the function $\alpha_{U}$ belongs to $\left(C_{0}, l^{1}\right)\left(\mathbf{R}^{a}\right)$.

Proof: . Since $\alpha_{U_{i}}(i=1, \ldots, a)$ is an even function we have for $n$ in $\mathrm{Z}-\{0,-1\}$ that

$$
\sup _{t \in[0,1]} \alpha_{U_{i}}(t+n)=\sup _{t \varepsilon[0,1]} \alpha_{U_{2}}(t-(1+n)) \leq \frac{2}{N_{i} \pi} \frac{1}{n^{2}} .
$$

If $n \varepsilon\{0,-1\}$, then there exists a constant $C_{i}$ such that

$$
\sup _{t \in[0,1]} \alpha_{U_{i}}(t+n) \leq \frac{N_{i}}{\pi} C_{i}
$$


because the limit

$$
\lim _{t \rightarrow-n} \frac{1-\cos N_{i}(n+t)}{\left(N_{i}(n+t)\right)^{2}}
$$

exists.

Therefore for all $i=1, \ldots, a$ and all integer $n$ we have that

$$
\sup _{t \in[0,1]}\left|\alpha_{U_{i}}(t+n)\right| \leq c a_{n}
$$

where

$$
c=\max _{1 \leq i \leq a}\left(2 /\left(N_{i} \pi\right), N_{i} C_{i} / \pi\right)
$$

and $a_{n}$ is equal to $1 / n^{2}$ if $n \varepsilon \mathbf{Z}-\{0,-1\}$, and to 1 if $n \varepsilon\{0,-1\}$.

Finally, for $i=1, \ldots, a$ we have that

$$
\begin{aligned}
\left\|\alpha_{U_{i}}\right\|_{\infty 1} & =\sum_{\mathbf{Z}} \sup _{t \varepsilon[n, n+1]}\left|\alpha_{U_{i}}(t)\right| \\
& =\sum_{\mathbf{Z}} \sup _{t \in[0,1]}\left|\alpha_{U_{i}}(t+n)\right| \\
& \leq C \sum_{\mathbf{Z}} a_{n}<\infty .
\end{aligned}
$$

From the definition of the norm \|\|$_{\infty 1}$, it is easy to see that

$$
\left\|\alpha_{U}\right\|_{\infty 1}=\Pi_{i=1}^{\alpha}\left\|\alpha_{U_{i}}\right\|_{\infty}
$$

Corollary 1.2. For each $U$ in $\mathcal{N}_{0}(G)$, the function $\varphi_{U}$ belongs to $\left(C_{0}, l^{1}\right)(G)$.

Proof: By (1) we have for all $(t, s)$ in $G$ that

$$
\varphi_{U}(t, s)=\alpha_{U}(t) \beta_{U}(s) \leq B_{U} \alpha_{U}(t),
$$

hence

$$
\left\|\varphi_{U}\right\|_{\infty 1} \leq B_{U} \sum_{n \varepsilon \mathbf{Z}^{a}} \sup _{t+n \varepsilon[0,1]^{a}}\left|\alpha_{U}(t)\right|=B_{U}\left\|\alpha_{U}\right\|_{\infty 1}
$$

For the rest of this paper $\varphi_{U}, \alpha_{U}$, and $\beta_{U}$ are as indicated in this section. 


\section{Main Theorem}

In this second section we want to prove that

$$
\lim _{U \rightarrow 0} \varphi_{U} * f(y)=f(y) \quad \text { almost everywhere }
$$

for all $f$ in $\left(L^{p}, l^{\infty}\right)(G)(2<p \leq \infty)$.

First, we prove two lemmas.

Lemma 2.1. Let $V$ and $K$ be two elements of $\mathcal{N}_{0}(G)$ of the form

$$
V=\left(-\delta_{1}, \delta_{1}\right) \times \cdots \times\left(-\delta_{a}, \delta_{a}\right) \times V_{H}
$$

and $K=\left[-\gamma_{1}, \gamma_{1}\right] \times \cdots \times\left[-\gamma_{a}, \gamma_{a}\right] \times K_{H}$, where $\delta_{i}>0, \gamma_{i}>0(i=$ $1, \ldots, a), V_{H}$ and $K_{H}$ are elements of $\mathcal{N}_{0}(G)$ contained in $H$, and $K_{H}$ is compact.

For $1 \leq p<\infty$, we define $\eta_{i}=\min \left(\delta_{i}^{2 p}, \gamma_{i}\right) \quad(i=1, \ldots, a)$, and we let $W_{H}$ be the interior of $K_{H}$. Then the set $W=\left[-\eta_{1}, \eta_{\mathrm{r}},\right] \times \cdots \times\left[-\eta_{a}, \eta_{a}\right] \times$ $W_{H}$ belongs to $\mathcal{N}_{0}(G)$ and for a fixed $y=\left(y_{0}, s_{0}\right)=\left(y_{1}, \ldots, y_{a}, s_{0}\right)$ in $G$, the element $W_{y}=y+W$ of $N_{y}(G)$ has the following properties:

2.1) $W_{y} \subseteq y+K_{H}$

2.2) If $\Pi_{a}=\left[-\eta_{1}+y_{1}, \eta_{1}+y_{1}\right] \times \cdots \times\left[-\eta_{a}+y_{a}, \eta_{a}+y_{a}\right]$, then

$$
\left[\int_{\Pi a} \alpha_{U}\left(y_{0}-x\right)^{p} d x\right]^{1 / p}=O\left(\Pi_{i=1}^{a} \delta_{i}\right)
$$

2.3) $\mathbf{R}^{a}-\Pi_{a} \subseteq \cup I_{n}$, where $\left\{I_{n}\right\}$ is a countable family of compact subsets of $\mathbf{R}^{a}$, and

$$
\sum_{\mathbf{N}}\left[\int_{I n} \alpha_{U}\left(y_{0}-x\right)^{p} d x\right]^{1 / p}=O\left(\Pi_{i=1}^{a} \delta_{i}\right) .
$$

2.4) There exists a constant $C$ such that $\sup _{\mathrm{N}} \mathcal{C}\left(I_{n}\right) \leq C$, where $\mathcal{C}\left(I_{n}\right)$ is the cordinality of the set

$$
\left\{j \varepsilon \mathbf{Z}^{a} \mid\left(j+[0,1]^{a}\right) \cap I_{n} \neq \phi\right\} .
$$

Proof: Several constants will appear during the proof and since their specific value is irrelevant for our needs we just write $C_{1}, C_{2}, \ldots C_{q}$. Part 
2.1) is clear. Set $J_{i}=\left[-\eta_{i}+y_{i}, \eta_{i}+y_{i}\right](i=1, \ldots, a)$. Part 2.2) follows from the continuity of $\alpha u_{i}$ because

(3)

$$
\left[\int_{J_{i}} \alpha_{U_{i}}\left(y_{i}-x\right)^{p} d x\right]^{1 / p}=\left[\int_{-\eta_{i}}^{\eta_{i}} \alpha_{U_{i}}(x)^{p} d x\right]^{1 / p} \leq C_{1} N_{i} \eta_{i}^{1 / p} \leq C_{2} \delta_{i} .
$$

Now, for each $i=1, \ldots, a$, let $L(n, i)$ and $R(n, i)(n \varepsilon \mathrm{N})$ be the intervals

$$
\left[-n-1-\eta_{i}+y_{i},-n-\eta_{i}+y_{i}\right] \text { and }\left[n+\eta_{i}+y_{i}, n+1+\eta_{i}+y_{i}\right]
$$

respectively. Then

$$
\mathbf{R}-J_{i}=\left(-\infty,-\eta_{i}+y_{i}\right) \cup\left(\eta_{i}+y_{i}, \infty\right) \subseteq \bigcup_{\mathbf{N}} L(i, n) \cup \bigcup_{\mathbf{N}} R(i, n),
$$

and

$$
\int_{L(n, i)} \alpha U_{i}\left(y_{i}-x\right)^{p} d x \leq C_{3} \delta_{i}^{p} a_{n}
$$

where

$$
a_{n}=\frac{1}{\left(n_{i}+n\right)^{2 p-1}}-\frac{1}{\left(n_{i}+n+1\right)^{2 p-1}} .
$$

Since $\sum a_{n}^{1 / p}$ converges we conclude that

$$
\sum_{\mathbf{N}}\left[\int_{L(n, i)} \alpha_{U_{i}}\left(y_{i}-x\right)^{p} d x\right]^{1 / p} \leq C_{4} \delta_{i}
$$

Similarly

$$
\sum_{N}\left[\int_{R(n, i)} \alpha_{U_{2}}\left(y_{i}-x\right)^{p} d x\right]^{1 / p}=C_{5} \delta_{i}
$$

Clearly $\sup _{\mathrm{N}} \mathcal{C}(L(n, i))$ and $\sup _{\mathrm{N}} \mathcal{C}(R(n, i))$ are less than or equal to 2 , hence for $i=1, \ldots, a$, the set $\mathbf{R}-J_{i}$ is equal to $\cup I_{n}$, where each $I_{n}$ is compact, $\sup \mathcal{C}\left(I_{n}\right) \leq 2$ and

$$
\sum_{\mathbf{N}}\left[\int_{I_{n}} \alpha_{U_{i}}\left(y_{i}-x\right)^{p} d x\right]^{1 / p}=O\left(\delta_{i}\right)
$$

Since $\mathbf{R}=\left(\mathbf{R}-J_{a}\right) \cup J_{a}$, and $J_{a}$ is compact, by (3) and (4) we see that $\mathbf{R}=\cup K_{n}$, with each $K_{n}$ compact, $\sup \mathcal{C}\left(K_{n}\right) \leq C_{6}$, and

$$
\sum_{N}\left[\int_{K_{n}} \alpha_{U_{a}}\left(y_{a}-x\right)^{p} d x\right]^{1 / p}=O\left(\delta_{a}\right)
$$


We prove properties 2.3) and 2.4) by induction on $a$. The case $a=1$ follows from (3). Suppose that 2.3) and 2.4) hold for $a-1$. That is, $\mathbf{R}^{a-1}-\Pi a-1 \subseteq \cup I_{n}$, each $I_{n}$ a compact subset of $\mathbf{R}^{a-1}, \sup C\left(I_{n}\right) \leq C_{7}$, and

$$
\sum_{N}\left[\int_{I_{n}} \Pi_{i=1}^{a-1} \alpha_{U_{i}}\left(y_{i}-x_{i}\right)^{p} d x\right]^{1 / p}=O\left(\Pi_{i=1}^{a-1} \delta_{i}\right) .
$$

By (4) with $i=a$, we have that $\mathbf{R}-J_{a} \leq U I_{j}$, each $I_{j}$ a compact subset of $\mathbf{R}, \sup \mathcal{C}\left(I_{j}\right) \leq 2$ and

$$
\sum_{\mathbf{N}}\left[\int_{I_{j}} \alpha_{U_{a}}\left(y_{a}-x\right)^{p} d x\right]^{1 / p}=O\left(\delta_{\alpha}\right) .
$$

Then

$$
\begin{aligned}
\mathbf{R}^{a}-\Pi a & =\left(\mathbf{R}^{a-1} \times \mathbf{R}\right)-(\Pi(a-1) \times J a) \\
& =\left(\mathbf{R}^{a-1}-\Pi(a-1)\right) \times(\mathbf{R} \cup \Pi(a-1)) \times(\mathbf{R}-J a) \\
& \leq \bigcup_{n, m}\left(I_{n} \times K_{m}\right) \bigcup_{\mathbf{N}}(\Pi(a-1) \times I j) .
\end{aligned}
$$

The sets $I_{n} \times K_{m}$ and $\Pi(a-1) \times I j$ are compact subsets of $\mathbf{R}$, for all $n, m, j$. Hence $\sup \mathcal{C}\left(I_{n} \times K_{m}\right) \leq C_{8}$ and $\sup \mathcal{C}(\Pi(a-1) \times I j) \leq C_{9}$. Therefore 2.4) holds with $C=\max \left(C_{8}, C_{9}\right)$. Finally, by (5) and (6) we have that

$$
\begin{aligned}
& \sum_{n, m}\left[\int_{I_{n} \times K_{m}} \alpha_{U}\left(y_{0}-x\right)^{p} d x\right]^{1 / p}= \\
& =\sum_{\mathbf{N}}\left[\int_{I_{n}} \Pi_{i=1}^{\alpha-1} \alpha_{U_{i}}\left(y_{i}-x_{i}\right)^{p} d x\right]^{1 / p} \sum_{\mathbf{N}}\left[\int_{K_{m}} \alpha_{U_{a}}\left(y_{a}-x\right)^{p} d x\right]^{1 / p}= \\
& =O\left(\Pi_{i=1}^{\alpha} \delta_{i}\right) .
\end{aligned}
$$

We conclude from (3) and (7) that

$$
\begin{aligned}
& \sum_{\mathbf{N}}\left[\int_{\Pi a-1 \times I j} \alpha_{U}\left(y_{0}-x\right)^{p} d x\right]^{1 / p}= \\
&=\Pi_{i=1}^{a-1}\left[\int_{I_{3}} \alpha_{U_{i}}\left(y_{i}-x\right)^{p} d x\right]^{1 / p} \sum_{\mathbf{N}}\left[\int_{I_{j}} \alpha_{U_{a}}\left(y_{a}-x\right)^{p} d x\right]^{1 / p}= \\
&=O\left(\Pi_{i=1}^{a} \delta_{i}\right) .
\end{aligned}
$$


Lemma 2.2. For each $V_{y}$ in $\mathcal{N}_{y}(G) \quad(y \varepsilon G)$

$$
\lim _{u \rightarrow 0} \int_{G-V_{y}} \varphi_{U}(y-x) f(x) d x=0
$$

for all $f$ in $\left(L^{p}, l^{\infty}\right)(G)(1<p \leq \infty)$.

Proof: Let $y=\left(y_{1}, \ldots, y_{a}, s_{0}\right)=\left(y_{0}, s_{0}\right)$ be an element of $\mathbf{R}^{a} \times G_{1}$. We choose two elements $V$ and $K$ of $\mathcal{N}_{0}(G)$ with the same form as in Lemma 2.2 , such that $y+K \subseteq V_{y}$ and $V \subseteq U$.

Following the notation of Lemma 2.2 , we set $\eta_{i}=\min \left(\delta_{i}^{2 p}, \gamma_{i}\right)(i=$ $\left.I_{1} \ldots, a\right)$, and $W_{H}$ the interior of $K_{H}$. Then the set $W=\left[-\eta_{1}, \eta_{1}\right] \times$ $\cdots \times\left[-\eta_{a}, \eta_{a}\right] \times W_{H}$ satisfies the properties listed in Lemma 2.2. Hence by property 2.1 ) it is enough to prove that

$$
\lim _{U \rightarrow 0} \int_{G-W_{y}} \varphi_{U}(y-x) f(x) d x=0 .
$$

Since

$$
G-W_{y}=\left(\mathbf{R}^{a}-\Pi_{a}\right) \times G_{1} \cup \Pi_{a} \times\left(G_{1}-\left(s_{0}+W_{H}\right)\right),
$$

we have by the definition of the function $\varphi_{U}$, that

$$
\varphi_{U}(y-x)=\alpha_{U}\left(y_{0}-t\right) \beta_{U}\left(s_{0}-s\right)=0
$$

if $s_{0}-s \not H$, and $x=(t, s)$ in $G$. Hence

$$
\begin{aligned}
\int_{G-W_{\mathbf{y}}} \varphi_{U}(y-x) f(x) d x & =\int_{\left(\mathbf{R}^{a}-\Pi a\right) \times\left(s_{0}+H\right)} \varphi_{U}(y-x) f(x) d x \\
& +\int_{\Pi a \times\left(s_{0}+\left(H-W_{g}\right)\right)} \varphi_{U}(y-x) f(x) d x .
\end{aligned}
$$

Let $\left\{I_{n}\right\}$ be the countable family of sets given by property 2.3 ). For each $I_{n}$ we have by the Hölder inequality and (1)

$$
\begin{aligned}
\int_{I_{n} \times\left(s_{0}+H\right)}\left|\varphi_{U}(y-x) f(x)\right| d x \leq\left\|f \chi_{I_{n} \times\left(s_{0}+H\right)}\right\|_{p} B_{U} \leq & \\
& \leq\left[\int_{I_{n}} \alpha_{U}\left(y_{0}-x\right)^{p^{\prime}} d x\right]^{1 / p^{\prime}}
\end{aligned}
$$


By property 2.4) $\sup _{\mathbf{N}}\left|S\left(I_{n} \times\left(s_{0}+H\right)\right)\right| \leq C$, where $C$ is a constant, and $\left|S\left(I_{n} \times\left(s_{0}+H\right)\right)\right|$ is the number of $K_{\alpha}$ 's (as defined in $[\mathbf{S}]$ ) such that $I_{n} \times\left(s_{0}+H\right) \cap K_{\alpha} \neq \phi$. This implies that for all $n \in \mathbf{N}$

$$
\left\|f \chi_{I_{n} \times\left(s_{0}+H\right)}\right\|_{p} \leq\left|S\left(I_{n} \times\left(s_{0}+H\right)\right)\right|\|f\|_{p \infty} \leq C|| f \|_{p \infty} .
$$

Thus, we conclude from 2.2 ) that

(9)

$$
\begin{aligned}
\int_{\left(\mathbf{R}^{a}-\Pi_{a}\right) \times G_{1}} \varphi_{U}(y-x)|f(x)| d x \leq & \\
\leq C\|f\|_{\infty} B_{U} \sum_{\mathbf{N}}\left[\int_{I_{n}} \alpha_{U}\left(y_{0}-x\right)^{p^{\prime}} d x\right]^{1 / p^{\prime}} & = \\
& =O\left(\Pi_{i=1}^{a} \delta_{i} B_{U}\right) .
\end{aligned}
$$

Applying Hölder's inequality we get

$$
\begin{aligned}
& \int_{\Pi a \times\left(s_{0}+\left(H-W_{H}\right)\right)} \varphi_{U}(y-x)|f(x)| d x \leq \\
& \left.\quad \leq B_{U}|| f \|_{p \infty}\right] S\left(\Pi a \times\left(s_{0}+\left(H-W_{H}\right)\right) \mid\left[\int_{\Pi a} \alpha_{U}\left(y_{0}-x\right)^{p^{\prime}} d x\right]^{p^{\prime}} .\right.
\end{aligned}
$$

Note that $\Pi a \times\left(s_{0}+\left(H-W_{H}\right)\right)$ is compact ( $H$ is compact and $H-W_{H}$ is closed), and because $B_{U} \rightarrow 0$ as $U \rightarrow 0$

Now, since $\Pi a \rightarrow y$ as $U \rightarrow 0$ and $s_{0}+\left(H-W_{H}\right) \leq s_{0}+H$ is independent of $U$, we have that $\mid S\left(\Pi a \times\left(s_{0}+\left(H-W_{H}\right) \mid \rightarrow 0\right.\right.$ as $U \rightarrow 0$. Therefore by property 2.2 )

$$
\int_{\Pi a \times\left(s_{0}+\left(H-W_{H}\right)\right)} \varphi_{U}(y-x)|f(x)| d x \rightarrow 0 \quad \text { as } \quad U \rightarrow 0 .
$$

The result follows from (8), (9), and (10).

Theorem 2.3. For all $f$ in $\left(L^{p}, l^{\infty}\right)(G), 2 \leq p \leq \infty$,

$$
\lim _{U \rightarrow 0} \int_{G} \varphi_{U}(y-x) f(x) d x=f(y)
$$

almost everywhere.

Proof: Let $V_{y}$ be in $N_{y}$ compact. We have to show, by Lemma 2.2, that

$$
\lim _{V \rightarrow 0} \int_{V_{y}} \varphi_{U}(y-x) f(x) d x
$$


converges to $f(x)$ almost everywhere. Since the function $f$ belongs to $\left(L^{p}, l^{\infty}\right) \subseteq\left(L^{2}, l^{\infty}\right)$, the function $g=f \chi_{V_{y}}$ belongs to $L^{2}(G)$, and by Corollary $1.2,1.1$ ), and 1.4) each $\varphi_{U}$ also belongs to $L^{2}(G)$. Hence by the Parseval identity, we have that

$$
\begin{aligned}
\int_{V_{y}} \varphi_{U}(y-x) f(x) d x & =\int_{G} \varphi_{U}(y-x) g(x) d x \\
& =\int_{\Gamma} \hat{\varphi}_{U}(\hat{x}) \hat{g}(-\hat{x}) \overline{[y, \hat{x}]} d \hat{x}
\end{aligned}
$$

By the Lebesque Dominated Convergence theorem (see properties 1.3 and 1.6) we have that

$$
\begin{aligned}
\lim _{U \rightarrow 0} \int_{V_{y}} \varphi_{U}(y-x) f(x) d x & =\lim _{U \rightarrow 0} \int_{\Gamma} \hat{\varphi}_{U}(\hat{x}) \hat{g}(-\hat{x}) \overline{[y, \hat{x}]} d \hat{x} \\
& =\int_{\Gamma} \hat{g}(-\hat{x}) \overline{[y, \hat{x}]} d \hat{x}=g(y)
\end{aligned}
$$

almost anywhere.

\section{Fourier Transform of Unbounded Measures}

The space $M_{q}(G)(1 \leq p<\infty)$ of unbounded measures of type $q[\mathbf{S}]$ : consists of Radon measures $\mu$ with finite norm $\left\{\mu \mu \|_{q}\right.$ given by $\left[\sum_{J}|\mu|\left(K_{\alpha}\right)^{q}\right]^{1 / q}$. If $G=\mathbf{R}$, then the family $\left\{K_{\alpha}\right\}$ can be taken as $\{[n, n+1] \mid n \varepsilon \mathbf{Z}\}$.

In this section we generalize to locally compact abelian groups, the following theorem due to $\mathrm{F}$. Holland $[\mathbf{H}]$.

Theorem 3.1. Let $1 \leq q \leq 2$ and $\mu \varepsilon M_{q}(\mathbf{R})$. Then as $N \rightarrow \infty$

$$
\frac{1}{\sqrt{2 \pi}} \int_{-N}^{N} e^{-i \pm t} d \mu(t)
$$

converges in the norm of $\left(L^{q^{\prime}}, l^{\infty}\right)$ to a function $\hat{\mu}$ and

$$
\int h(x) \hat{\mu}(x) d x=\int \hat{h}(x) d \mu(x) \quad\left(h \varepsilon\left(L^{q}, l^{1}\right)(\mathbf{R})\right) .
$$

Further

$$
\sqrt{2 \pi} \hat{\mu}(x)=(C .1) \int e^{-i x t} d \mu(t)
$$


almost everywhere.

(C.1) means that the integral on the right is summable by the Cesáro method of order 1 to the value $\sqrt{2 \pi} \hat{\mu}(x)$.

It is easy to see, that for any measure $\mu$ in $M_{q} \quad(1 \leq q \leq 2)$, there is a net $\mu_{\alpha}$ of bounded measures such that $\lim \left\|\mu_{\alpha}-\mu\right\|_{q}=0$, and therefore by [S. Theorem 4.2] $\lim \left\|\hat{\mu}_{\alpha}-\hat{\mu}\right\|_{q \infty}=0$. This generalizes the first part of the theorem.

Theorem 3.2. Let $\mu$ be an element of $M_{q} \quad(1 \leq q \leq 2)$

i) $\int_{\Gamma} \overline{f(\gamma)} \hat{\mu}(\gamma) d \gamma=\int_{G} \bar{f}(x) d \mu(x)$ for all $f$ in $\left(L^{q}, l^{1}\right)(\Gamma)$.

ii) (C.1) $\int_{G} \overline{\gamma(x)} d \mu(x):=\lim _{U \rightarrow 0} \int_{G} \overline{\varphi_{U}(x) \gamma(x)} d \mu(x)=\hat{\mu}(\gamma)$ almost everywhere.

(C.1) means that the integral on the right is summable by the Cesaro method of order 1 to the value $\hat{\mu}(\gamma)$.

Proof: Let $\mu_{\alpha}$ be the net of bounded measures related to $\mu$, as mentioned above.

Since $\left(L^{q}, l^{1}\right)$ is a subspace of $L^{1}[\mathbf{S},(3,4)]$, we have by the Extended Parseval Formula [S. Lemma 4.1] that for any $f$ in $\left(L^{q}, l^{1}\right)(\Gamma)$,

$$
\int_{\Gamma} \overline{f(\gamma)} \mu_{\alpha}(\gamma) d \gamma=\int_{G} \overline{f(x)} d \mu_{\alpha}(x)
$$

By the Hölder inequality

$$
\begin{aligned}
& \int_{\Gamma}|f(\gamma)|\left|\hat{\mu}_{\alpha}(\gamma)-\hat{\mu}(\gamma)\right| d \gamma \leq \\
& \leq \sum_{J}\left[\int_{K_{\beta}}|f(\gamma)|^{q}\right]^{1 / q}\left[\int_{K_{\beta}}\left|\hat{\mu}_{\alpha}(\gamma)-\hat{\mu}(\gamma)\right|^{q^{\prime}} d \gamma\right]^{1 / q^{\prime}} \leq \\
& \leq\|f\|_{q 1}\left\|\hat{\mu}_{\alpha}-\hat{\mu}\right\|_{q^{\prime} \infty} .
\end{aligned}
$$

Similarly

$$
\int_{G}|\check{f}(x)| \quad d\left|\mu_{\alpha}-\mu\right|(x) \leq\|\check{f}\|_{\infty q^{\prime}}\left\|\mu_{\alpha}-\mu\right\|_{q} .
$$

Therefore the left side of (ii) converges to $\int_{\Gamma} \overline{f(\gamma)} d \mu(\gamma)$, and the right side to $\int_{G} \check{f}(x) d \mu(x)$. This proves $\left.\mathrm{i}\right)$.

By proposition 1.1 and $[\mathrm{S},(3.1)]$, each $\varphi_{U}$ belongs to $\left(L^{q}, l^{1}\right)(\Gamma)$, so from i)

$$
\int_{\Gamma} \varphi_{U}(y-\gamma) \dot{\mu}(\gamma) d \gamma=\int_{G} y(x) \overline{\hat{\varphi}_{U}(x)} d \mu(x)
$$

Hence, part ii) follows from Theorem 2.3 . 


\section{References}

[AGL] L. Argabright and J. Gil de Lamadrid, Fourier analysis of unbounded measures on locally compact abelian groups, Memoirs Am. Math. Soc. 145 (1974).

[F] H. G. Feichtinger, On a new segal algebra, Mh. Math. 92 (1981), 269-289.

[H.] F. HOLLAND, Harmonic analysis on amalgams of $L^{p}$ and $l^{q}, J$. London Math. Soc. (2) 10 (1975), 295-305.

[L] V. LOSERT, A characterization of the ninimal strongly character invariant segal algebra, Ann. Inst. Fourier 30, 3 (1980), 129-139.

[Si] A. B. SIMON, Cesáro summability on groups: Characterization of Fourier transforms, Function Algebras. Proc. Internat. Sypos. on Function Algebras, Tulane Univ. (1965).

[S] J. STEWART, Fourier transforms of unbounded measures, Can. J. Math. 31, no. 6 (1979), 1281-1292.

[TS] TORRES DE SQUiRe, Multipliers for amalgams and the algebra $S_{0}(G)$, Can. J. Math. 39, no. 1 (1987), 123-148.

[W] N. WIENER, "The Fourier integral and certain of its applications," Cambridge Univ. Press, 1958.

University of Regina

Regina, Saskatchewan

S4S 0A2

CANADA

Primera versio rebuda el 8 d'Abril de 1991 , darrera versió rebuda el 8 de Setembre de 1992 